\title{
Editorial
}

\section{Pulvinar Complex: Relay Center or Something More?}

\author{
Jaikishan Jayakumar, PhD* \\ Center for Computational Brain Research, Indian Institute of Technology-Madras, Chennai,Tamil Nadu 600036, India
}

\section{*Corresponding author}

Jaikishan Jayakumar, PhD

Center for Computational Brain Research, Indian Institute of Technology-Madras, Chennai,Tamil Nadu 600036, India; E-mail: Jaikishan.jayakumar@gmail.com

\author{
Article information \\ Received: July $2^{\text {nd }}, 2018$; Accepted: July $26^{\text {th }}, 2018$; Published: July $30^{\text {th }}, 2018$
}

\section{Cite this article}

Jayakumar J. Pulvinar complex: Relay center or something more? Neuro Open J. 2018; 5(I): el-e2. doi: 10.17/40/NOJ-5-e008

$\mathrm{T}$ he pulvinar is a collection of thalamic nuclei which is often considered mainly with visual processing and attention. The unique thing about this thalamic brain area is that, in phylogeny, this area is considered to be much more advanced in primates and virtually absent in rodents ${ }^{1}$, making it an important brain area to understand in human brain evolution. This editorial aims to shed light on the structure and function of the pulvinar.

The pulvinar that is described in this article mainly represents the pulvinar of primates including humans as they are much more defined and developed compared with lesser species. Based on the cyto- and myeloarchitecture, the pulvinar can be divided into medial, lateral, inferior and the more anterior oral pulvinar $^{2}$. This classification, however, is purely anatomical and controversial and does not represent the functional classification of the nuclei. It is still unclear as to how many nuclei there are in the various divisions of the pulvinar. The majority of visual zones within the pulvinar for e.g. are found in the lateral and the inferior zones. Within the visual pulvinar, there is at least three subdivisions that have been named based on Calbindin staining ${ }^{3}$. In addition, the visual pulvinar is also classified into two zones depending on its recipients: a striate cortical zone and a superior collicular zone ${ }^{4}$.

The pulvinar is unique from other sensory nuclei within the thalamus in that it receives a majority of its inputs from the cortical structures rather than sensory structures for e.g. lateral geniculate nucleus is driven by the retina. Moreover, functionally the pulvinar looks to "mimic" the topographical organization of the cortical structures from which it receives input. This has led to the name "associative nuclei" rather than sensory or motor nuclei for this area.
The non-visual areas of the pulvinar which is mainly the medial pulvinar is involved in emotional salience estimation ${ }^{6}$ and has extensive connections with many paralimbic cortical structures and higher multisensory cortex ${ }^{7}$. In addition, this area has strong connections with emotional centers such as the amygdala ${ }^{8}$.

As mentioned before, the lateral and the inferior zones is mainly visual except for a small area called as the lateral posterior pulvinar ${ }^{9}$ which is thought to be involved in motor salience. The visual part of the pulvinar, particularly the striate cortex recipient zones are topographically aligned to their inputs from visual areas such as V1, V2, V5 as well as higher areas across both the dorsal visual stream and the ventral visual stream. This results in the possibility of this area being a point of interaction between the two streams and thus can effectively help in visual attention. The pulvinar is theorized to promote large scale synchronization across different networks within the cortex ${ }^{10}$. In addition, the pulvinar also enhances visual processing within the cortex by synchronizing gamma band oscillations within the pulvino-cortical networks ${ }^{11}$. In addition, a more recent study by Saalmann et al., ${ }^{12}$ shows that the pulvinar demonstrates alpha band synchronization that serves as an attentional communication mechanism. Using behavioral task of visual discrimination, they were able to show the synchronization of two different cortico-thalamic systems namely area 17 and the ventromedial region of the caudal part of the lateral zone of the pulvinar and the other network being area 18 and the dorsolateral part of the same region of the pulvinar.

The pulvinar has also been shown to be involved in "distractor filtering"13,14, contrast processing ${ }^{15}$, and interhemispheric integration ${ }^{16}$. The dorsal aspect of the pulvinar 
nucleus is also a critical hub for spatial attention and selection of visually guided actions ${ }^{17}$.

There is also evidence ${ }^{18}$ that a subset of nuclei in the medial part of the inferior pulvinar act as a subcortical component of the dorsal stream and another subset in the lateral part of the inferior pulvinar and the neighboring ventrolateral nucleus of the lateral pulvinar are part of the ventral stream of processing.

In summary, the pulvinar can be considered not as a simple relay center but as a thalamic brain area that serves as an integrator for most visual processing areas through possibly a mechanism of intra brain oscillations. Although its function as a visual processing area is somewhat clear how it relates to other modalities and intermodality synchronization is still unclear and why this area, in an evolutionary timeframe, has evolved in primates still remains a mystery.

\section{REFERENCES}

1. Chalfin BP, Cheung DT, Muniz JA, de Lima Silveira LC, Finlay BL. Scaling of neuron number and volume of the pulvinar complex in New World primates: Comparisons with humans, other primates, and mammals. J Comp Neurol. 2007; 504(3): 265274. doi: $10.1002 /$ cne. 21406

2. Shipp S. Pulvinar Structure and Circuitry in Primates. Encyclopedia of Neuroscience. London, UK: Elsevier; 2009: pp. 1233-1244.

3. Cusick CG, Scripter JL, Darensbourg JG, Weber JT. Chemoarchitectonic subdivisions of the visual pulvinar in monkeys and their connectional relations with the middle temporal and rostral dorsolateral visual areas, MT and DLr. J Comp Neurol. 1993; 336(1): 1-30. doi: 10.1002/cne.903360102

4. Felleman DJ. Visual System in the Brain in International Encyclopedia of the Social \& Behavioral Sciences. NY, USA: Elsevier; 2001: pp. 16278-16285.

5. Sherman SM, Guillery RW. Functional organization of thalamocortical relays. J Neurophysiol. 1996; 76: 1367-1395. doi: 10.1152/jn.1996.76.3.1367

6. Padmala S, Lim SL, Pessoa L. Pulvinar and affective significance: Responses track moment-to-moment stimulus visibility. Front Hum Neurosci. 2010; 4: 64. doi: 10.3389/fnhum.2010.00064

7. Mufson EJ, Mesulam MM. Thalamic connections of the insula in the rhesus monkey and comments on the paralimbic connectivity of the medial pulvinar nucleus. J Comp Neurol. 1984; 227(1): 109-

\section{0. doi: 10.1002/cne. 902270112}

8. Rosenberg DS, Mauguière F, Demarquay G, Ryvlin P, Isnard J, Fischer C, Guénot M, Magnin M. Involvement of medial pulvinar thalamic nucleus in human temporal lobe seizures. Epilepsia. 2006; 47(1): 98-107. doi: 10.1111/j.1528-1167.2006.00375.x

9. Sherman SM, Guillery RW. Distinct functions for direct and transthalamic corticocortical connections. J Neurophysiol. 2011; 106(3): 1068-1077. doi: 10.1152/jn.00429.2011

10. Shipp S. The functional logic of cortico-pulvinar connections. Philos Trans R Soc Lond B Biol Sci. 2003; 358(1438): 1605-1624. doi: 10.1098/rstb.2002.1213

11. Shumikhina S, Molotchnikoff S. Pulvinar participates in synchronizing neural assemblies in the visual cortex, in cats. Neurosci Lett. 1999; 272(2): 135-139.

12. Saalmann YB, Pinsk MA, Wang L, Li X, Kastner S. The pulvinar regulates information transmission between cortical areas based on attention demands. Science. 2012; 337(6095): 753-756. doi: 10.1126/ science. 1223082

13. Fischer J, Whitney D. Attention gates visual coding in the human pulvinar. Nat Commun. 2012; 3: 1051. doi: 10.1038/ncomms2054

14. Strumpf H, Mangun GR, Boehler CN, et al. The role of the pulvinar in distractor processing and visual search. Hum Brain Mapp. 2013; 34(5): 1115-1132. doi: 10.1002/hbm.21496

15. Cortes NH, Van Vreeswijk C. The role of pulvinar in the transmission of information in the visual hierarchy. Front Comput Neurosci. 2012; 6: 29. doi: 10.3389/fncom.2012.00029

16. Shinomoto S, Kim H, Shimokawa T, et al. Relating neuronal firing patterns to functional differentiation of cerebral cortex. PLoS Computational Biology. 2009; 5(7): e1000433. doi: 10.1371/ journal.pcbi.1000433

17. Wilke M, Turchi J, Smith K, Mishkin M, Leopold DA. Pulvinar inactivation disrupts selection of movement plans. Journal of Neuroscience. 2010; 30(25): 8650-8659. doi: 10.1523/ JNEUROSCI.0953-10.2010

18. Kaas JH, Lyon DC. Pulvinar contributions to the dorsal and ventral streams of visual processing in primates. Brain Res Rev. 2007; 55(2): 285-296. doi: 0.1016/j.brainresrev.2007.02.008 